\title{
Black cohosh (Cimicifuga racemosa) is a non-estrogenic alternative to hormone replacement therapy
}

\author{
Wolfgang Wuttke* and Dana Seidlová-Wuttke
}

\begin{abstract}
Hormone replacement therapy is still a popular and most effective treatment for vasomotor symptoms and bone loss prevention in the postmenopause but it is not without risks. This has driven many climacteric women to seek for alternatives, chiefly natural products. Phytoestrogens containing soy or red clover preparations, however, when taken at the recommended daily doses, proved to be ineffective to ameliorate climacteric complaints and to prevent osteoporosis. Cimicifuga racemosa (CR) preparations, on the other hand, have been shown to ease climacteric distress. There is a widespread, but false, belief that the efficacy of CR preparations is linked to the presence of phytoestrogens in the plant. This review aims at summarizing the available in vitro and in vivo evidence showing that compounds in CR extracts do not bind to oestrogen receptors and thus do not exert any estrogenic effects in the uterus and mammary gland, as shown in vivo in experiments on ovariectomized rats and clinically in postmenopausal women. Studies in ovariectomized rats and in women suffering from climacteric complaints have indicated that substances with neurotransmitter-like activities affect beneficially postmenopausal symptoms such as hot flushes. Some of these compounds, such as actein-like triterpenes with GABA-ergic activity and a serotonin analogue, are present and have been identified in the CR extracts. We conclude that these activities explain most likely the beneficial effects of CR extracts on climacteric complaints.
\end{abstract}

Keywords: Black cohosh; Cimicifuga racemosa; Hormone replacement therapy; Climacteric complaints; Menopause; Non-oestrogenic effects

\section{Review}

Hormone replacement therapy (HRT) is still the most effective treatment of menopausal symptoms, providing also some help in the prevention of osteoporosis. Fastidious or serious side effects however, limit its use. HRT has been shown, for instance, to increase mammary cancer [1-5] and in the Women's Health Initiative (WHI) study the administration of a combination of conjugated estrogens with medroxyprogesterone acetate over more than 8 years resulted in an increased risk for lethal cardiovascular events [1], which led to its premature termination. Such events actually occur only when HRT is started late in the postmenopause, whereas it may in fact protect against cardiovascular diseases when immediately started at the onset of the menopause [6].

\footnotetext{
* Correspondence: w.wuttke@verdevital.de

Hormone and Obesity Center Göttingen, Bahnhofsallee 1d, D-37081 Göttingen, Germany
}

Women are looking for HRT substitutes to ameliorate climacteric complaints, particularly hot flushes. The ideal products would not be estrogenic in the uterus and in the mammary gland and augment blood clotting. Herbal remedies are increasingly recognized as an alternative to hormones and physicians and patients are willing to use them. There is also clinical evidence that selected herbal medicinal products may exert positive effects in some of the conditions typical of the menopausal period.

Far East Asian women, who traditionally eat large amounts of soy, develop breast cancer less often than Caucasian women: when Japanese women, however, migrate to the US and change their dietary habits, their female children develop mammary cancers at frequencies observed in the Caucasian population. This has generated the belief that soy, possibly because of the estrogenic isoflavones it contains, may prevent mammary cancer. In fact, in adult rats, a reduction of 7,12-dimethylbenz(a)anthracene

\section{实 Springer}




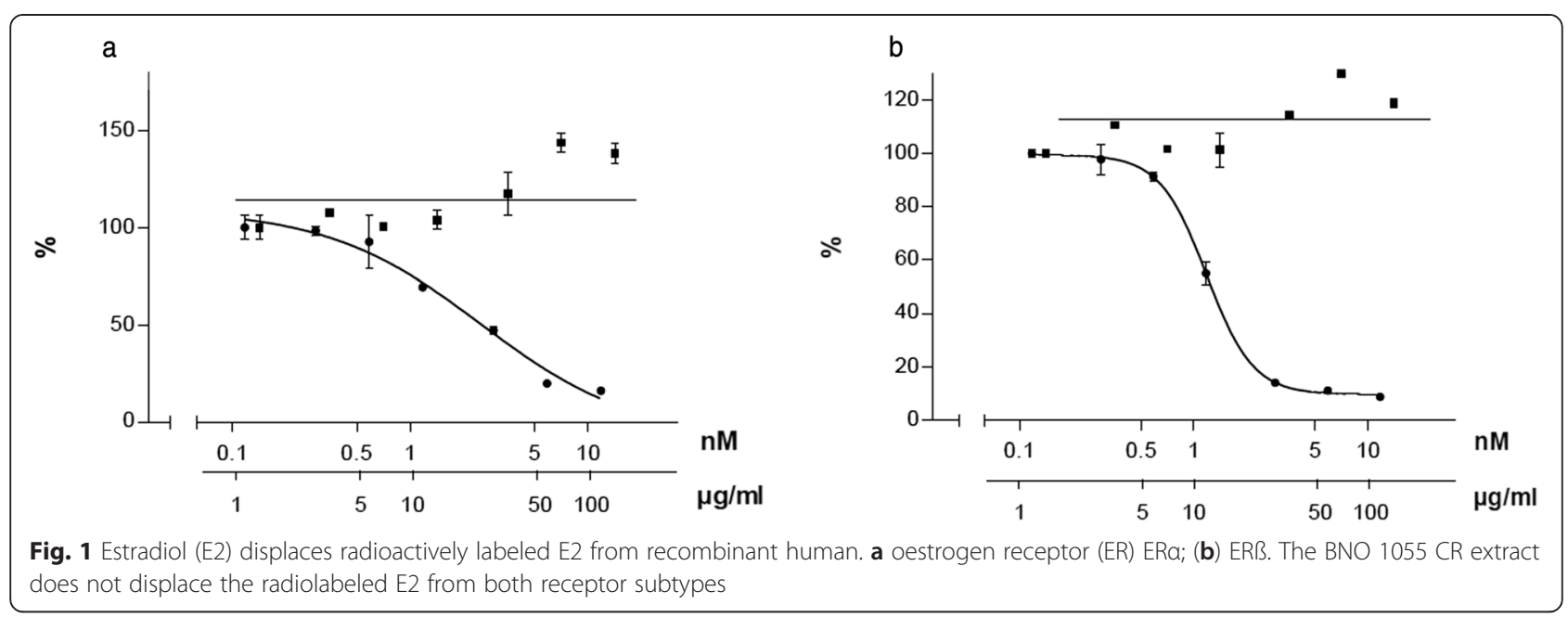

(DMBA)-induced mammary cancers by more than $50 \%$ was achieved by the isoflavone genistein, but only when the soy isoflavone was administered at peripubertal time $[7,8]$. The authors of these studies have suggested that early events are crucial for cancer protection and this has been confirmed in several other studies that have also elucidated the underlying molecular mechanisms [9]. There is now increasing evidence that this applies also to humans: In fact, recent studies have shown that Japanese girls who migrated after puberty to the US developed significantly less mammary carcinomas than those who went to the US before puberty or their Caucasian counterparts [10].

Soy or red clover preparations containing estrogenic isoflavones have been intensively investigated, but in most double blind, placebo controlled clinical studies carried out using the recommended daily doses of $50 \mathrm{mg}$ isoflavones these products had no significant effects on climacteric complaints [11-14] and no bone protective effects could be observed [13, 15, 16]. At this dosage the isoflavones did not exert any effects on the mammary gland and the uterus; on the other hand, 3-4-fold higher amounts of the daily recommended dose, often used by women in the hope of obtaining a better efficacy, may exert undesired estrogenic effects.

\section{Cimicifuga racemosa (black cohosh)}

Non-hormonal treatment with natural remedies may be an option and Cimicifuga racemosa (CR; synonym: Actaea racemosa) is probably the most promising available alternative. Its clinical efficacy and safety in climacteric symptoms have been evaluated in several studies with mainly positive outcomes. Traditionally, $\mathrm{CR}$ has been used by indigenous American Indians for aching muscles and joints, neuralgias, rheumatoid arthritis, menstrual cramps and more general gynecological complaints. CR originates from the US and it is now grown systematically in Europe. CR extracts have been marketed since 1956 in Germany and the scientific knowledge on this herbal medicine has been summarized in several monographs, among them and prominently the ESCOP monograph [17], leading to the publication of the Community Herbal Monograph on Cimicifuga racemosa (L.) Nutt. rhizoma by the EMA Herbal Medicinal Products Committee [18]. Preparations on the food supplement market containing Asian Cimicifuga species that have never been clinically tested and may contain quite different compounds are not covered by the Community Monograph and cannot be considered similar to the CR extracts.

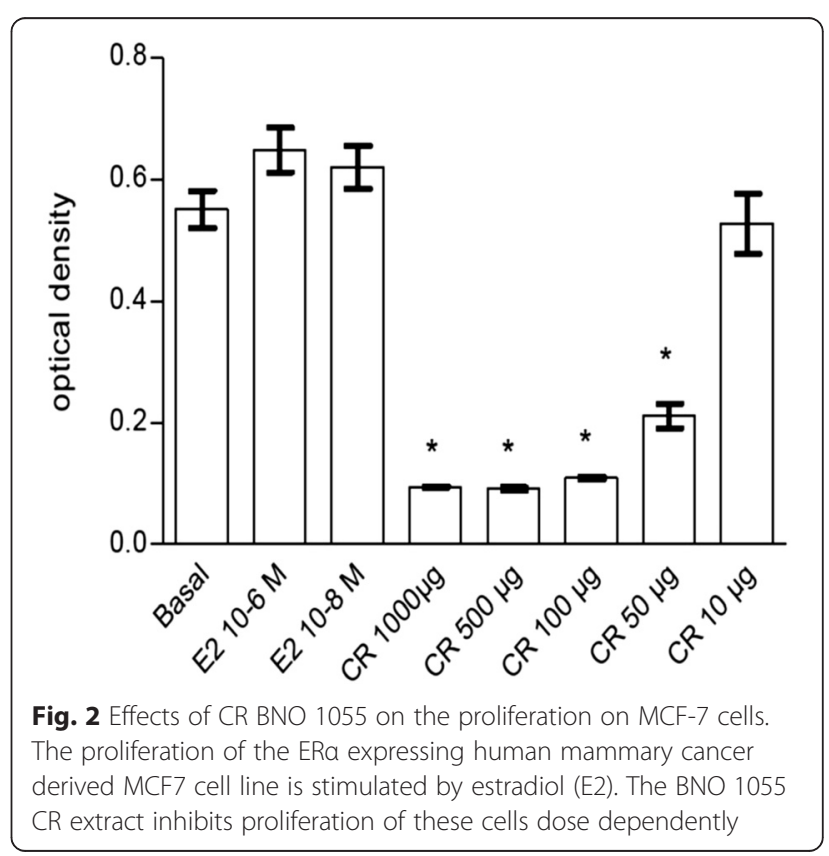




\section{Cimicifuga racemosa has no estrogenic activity and does not bind to estrogen receptors (ER)}

Almost thirty years ago an isopropanolic extract of $C R$ was described to contain the estrogenic isoflavone formononetine [19] but subsequent studies on a variety of CR extracts did not confirm this finding. In silico, in vitro and in vivo animal and clinical evidence, gathered mainly in experiments carried out with the aqueous/ethanolic CR extract BNO 1055", indicates that all CR extracts tested so far contain no estrogenic compounds.

Estrogenic compounds should bind to estrogen receptors (ER). Both ER $\alpha$ and ERß have been cloned and used in in vitro binding studies with the BNO 1055 CR extract [20]. No compound contained in the extract bound to the recombinant ER $\alpha$ or ERß (Fig. 1a, b).

Estrogenic compounds stimulate the proliferation of estrogen receptive human mammary carcinoma cells. In the MCF-7 carcinoma cell line, the most widely used cell line with estrogen reception properties, the proliferation is inhibited rather than stimulated by an isopropanolic extract of CR [21-23] or by the BNO 1055 CR extract [20] (Fig. 2). Mammary gland tissue and MCF-7 cells express aromatases that can increase the availability of estrogens in mammary glands by aromatizing androgens into estrogens. This conversion was inhibited by an ethanolic CR extract in MCF 7 cells [24], which would suggest some mammary cancer protective effects of the extract.

Effects by $\mathrm{CR}$ in the mammary gland and the uterus were tested in ovariectomized (ovx) rats, an OECD recommended model for the study of putatively estrogenic effects [25]. Animals were treated orally with the BNO 1055 CR extract for 3 months. Control animals received either sham or estrogen treatment. The extract did not affect the lobuloalveolar and duct apparatus of the ovx rats whereas estradiol (E2) containing food resulted in a
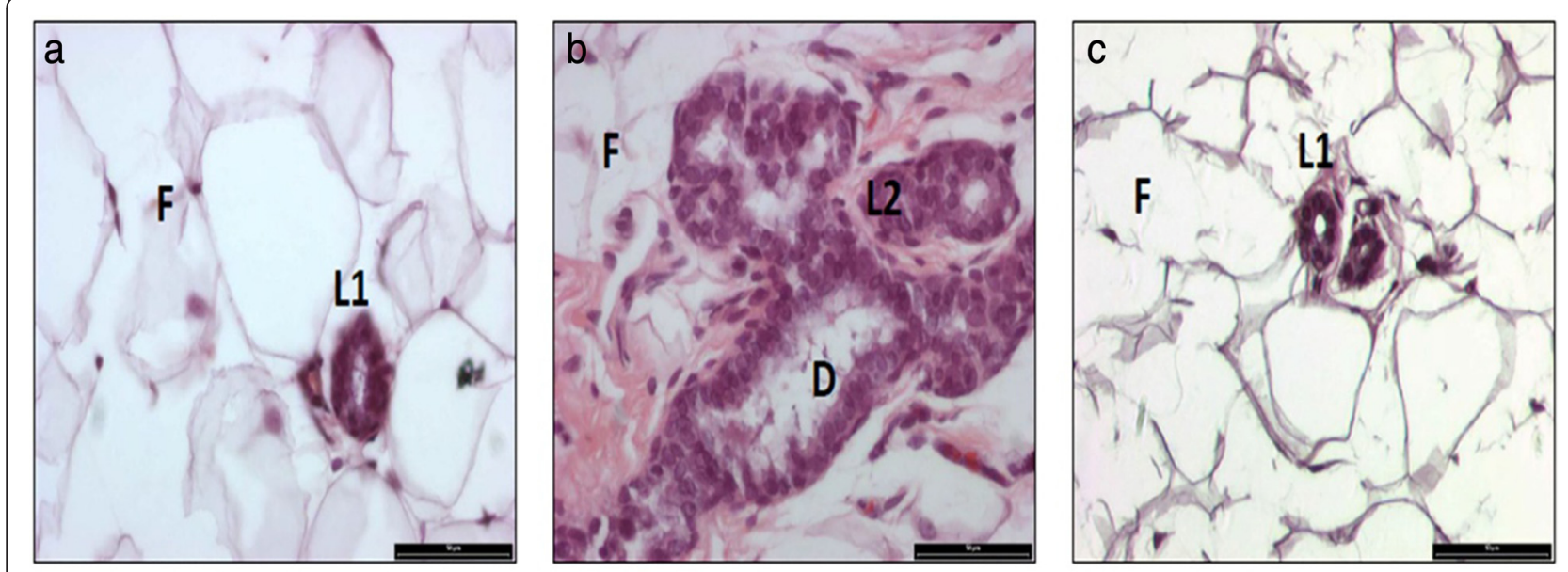

d

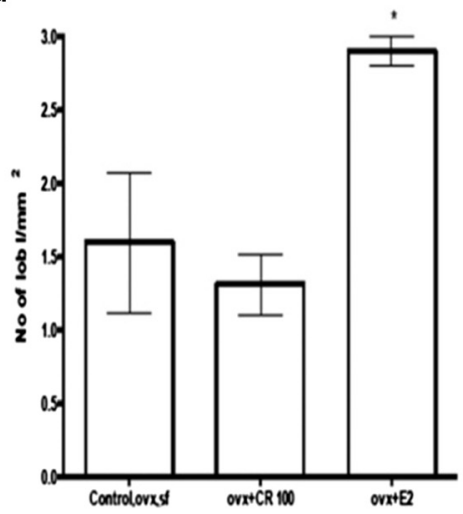

e

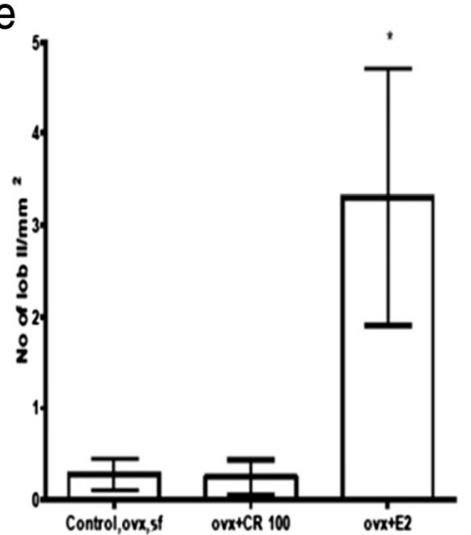

f

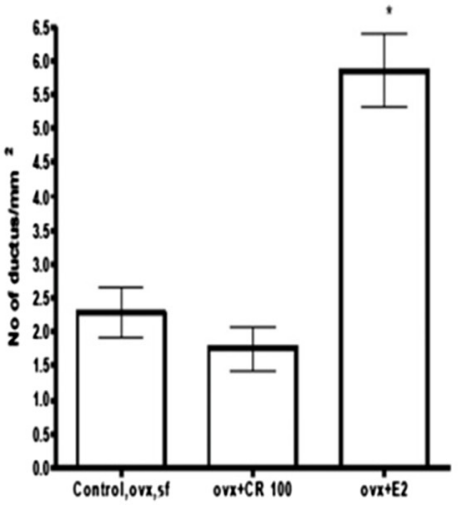

Fig. 3 a-c Histological appearance of mammary glands of (a) control ovx, (b) ovx + E2, (c) ovx + CR BNO 1055. Estradiol (E2) stimulated the number of lobuli in the mammary glands (b), but not in the sham (a) and in the BNO 1055 CR extract treated animals (c). D: duct; F: fat; L1: Lobulus 1; L2: Lobulus 2. d-f Histomorphometric evaluation of the histologic preparations after 3 months per food in ovx rats. $\mathbf{d}$ Effects of E2 and CR on number on lobulus $1 / \mathrm{mm}^{2}$; (e) Effects of E2 and CR on lobulus 2/. $\mathrm{mm}^{2}$; (f) Effects of E2 and CR on number of ducts/mm². E2 yielded a significant stimulation of lobuli type 1 (d) and 2 (e) and ducts (f) ( ${ }^{*} p<0.05$ vs control, ovx). CR: Cimicifuga racemosa BNO 1055; E2: Estradiol; lob: Lobulus; ovx: ovariectomised; sf: soy free food ( ${ }^{*} p<0.05$ vs. sham and vs. CR treated) 
massive stimulation of the lobuli and duct (Fig. 3a-f) [26]. Sprague-Dawley rats treated with DMBA developed typical mammary tumor structures that were inhibited by oral administration of an isopropanolic CR extract [27].

In a large, 6-month, clinical study in early postmenopausal women, BNO 1055 did not affect mammary gland density, as determined by mammography, or endometrial thickness [28]. An isopropanolic CR extract tested in 65 women who underwent mammography and fine needle aspiration biopsies of the breast did not increase mammographic breast density or cell proliferation [29]. In a prospective, observational study carried out in 50 breast cancer patients under Tamoxifen treatment therapy, an isopropanolic CR extract administered during 6 months reduced significantly psycho-vegetative symptoms as measured by the Menopause Rating Scale (MRS) [30]. A recently published case control study involving 949 breast cancer patients demonstrated that the use of several black cohosh preparations had significant breast cancer protective effects [31]. Finally, the effects of the intake of $2.5 \%$ triterpenes derived from CR extracts were compared to those of an extract containing trace amounts of triterpenes and no specific estrogenic effects on the breast were observed [32].

The evidence gathered so far suggests, therefore, a protective rather than a harmful effect on the mammary gland.

The uterine weight of ovx rats increases in response to an estrogenic stimulus [25]. In studies utilizing this animal model several CR extracts did not cause any increase in uterine weight [20]. Uterine histology and the expression of estrogen-regulated genes in the uterus remained unaffected, while treatment with E2 exerted typical estrogenic effects (Fig. 4a, b) [33].

In a placebo-controlled, clinical study the BNO 1055 CR extract had no effects on the endometrium, as opposed to an estrogen preparation that stimulated the increase of the endometrium thickness as measured by vaginal ultrasound [34]. In the longest clinical safety study ever conducted with CR, the same CR preparation was tested for one year and endometrial safety assessed by histological evaluation of the tissue. No case of endometrial abnormality was detected in more than 300 tested patients [28].

\section{Efficacy of CR in climacteric complaints}

Ovariectomised rats suffer from hot flushes [35-38] that occur as frequently as the LH pulses. Kapur et al. [39] have shown that the skin temperature of ovx rats increases at regular intervals (Fig. 5) and as a result of these pulses the mean skin temperature of ovx rats is significantly higher than in the intact individuals. Very likely the overactive pulse generator produces hot flushes also in ovx rats, similarly as in postmenopausal women. An effect of the aqueous-ethanolic BNO 1055 CR extract and of an isopropanolic CR extract on hot flushes in ovx rats has been demonstrated $[39,40]$. Ovx rats had a higher skin temperature than animals treated with the BNO 1055 CR extract (Fig. 6). It can therefore be safely concluded that CR extracts do not reduce hot flushes in ovx rats through estrogenic but rather through non-estrogenic, neurotransmitter-like substances.

\section{Clinical studies}

Several clinical trials have reported that black cohosh extracts reduce hot flushes. Most of these studies, however,
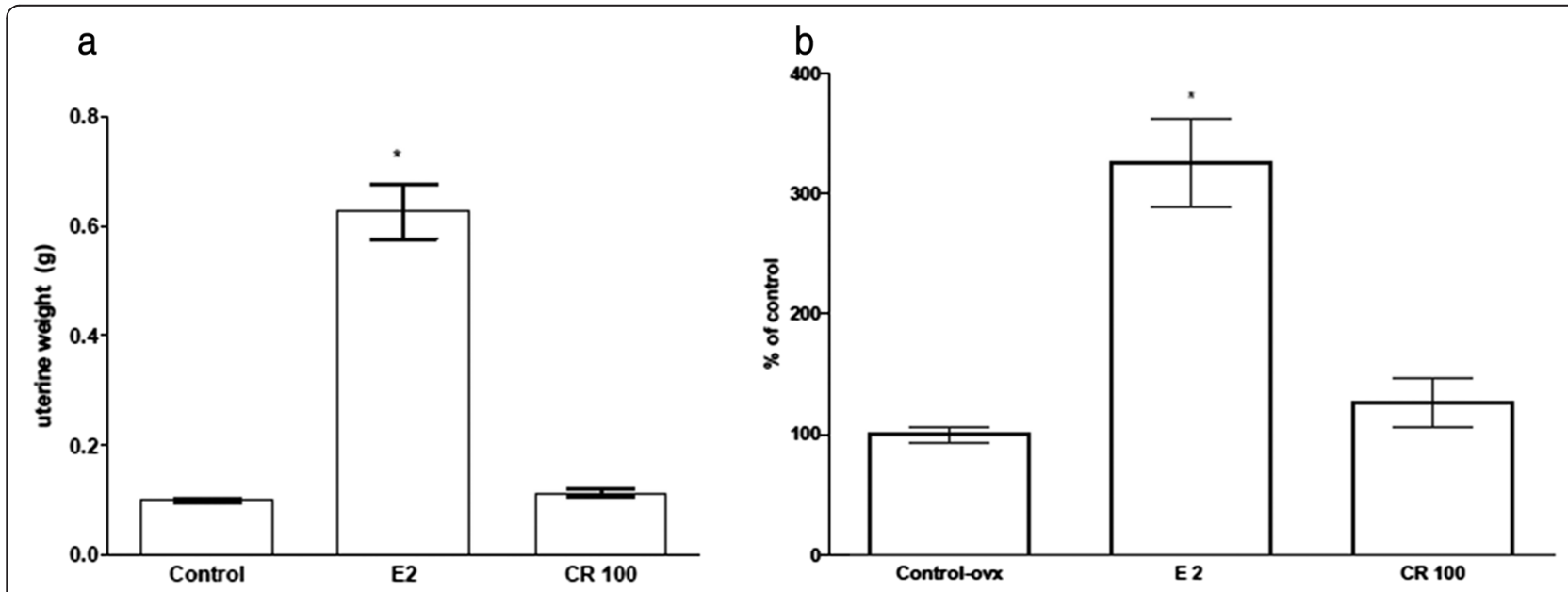

Fig. 4 Effects of E2 and CR BNO 1055 (12 weeks p.o.) in ovx rats on (a) uterine weight and (b) gene expression of IGF-1 in uterus. Uterine weights (a) and insulin like growth factor 1 (IGF1) gene expression (b) of ovx rats were significantly stimulated by E2. The stimulatory effect of E2 on uterine weights is partially mediated by E2-stimulated insulin like growth factor gene (IGF 1$)\left({ }^{*} p<0.05\right.$ vs Control and vs. CR 100). CR: Cimicifuga racemosa; E: Estradiol; IGF: insulin like growth factor; ovx: ovariectomised 


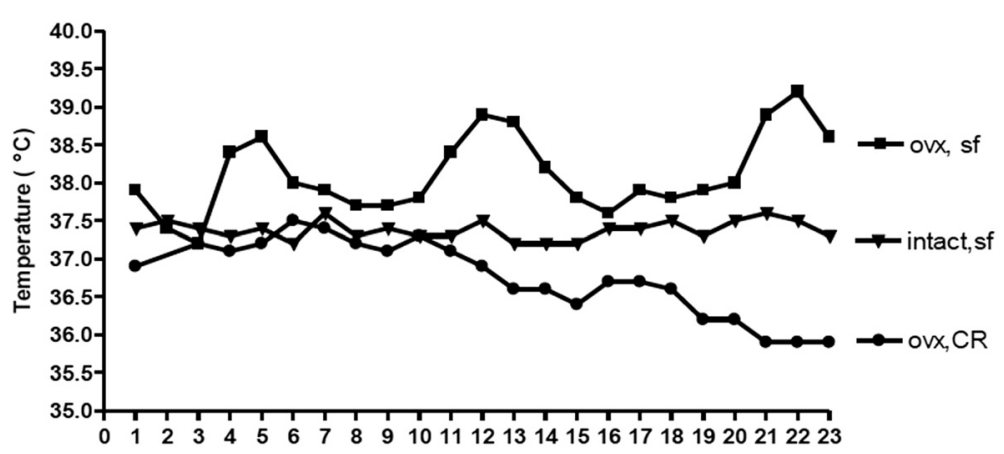

Fig. 5 Skin temperature of ovx and intact rats. Typical fluctuations of subcutaneously measured skin temperature in an ovx rat are not seen in intact or BNO 1055 CR extract treated animals (Scans every 5 min; n ovx, sf; intact, sf; Ÿ ovx). CR: Cimicifuga racemosa; ovx: ovariectomised; sf: soy free food

were open and uncontrolled or used too high extract dosages. A number of well-performed, double blind, placebo controlled studies, on the other hand, have demonstrated the beneficial effects of extracts of the CR rhizome on climacteric complaints.

Fourteen randomized, placebo or comparator controlled trials investigating various climacteric symptoms were identified (Tables 1-3). All trials using preparations of European origin and sold exclusively in pharmacies yielded positive results, with statistically significant improvement of climacteric complaints [30, 41-50]. In all but three studies either an isopropanolic or an aqueous/ ethanolic extract of $40 \mathrm{mg}$ of the dried plant rhizome (an amount equivalent to $4-8 \mathrm{mg}$ of dry extract) were used. On the other hand, four (three US and one
Australian) studies using much higher extract doses (up to $160 \mathrm{mg}$ of dry extract, equivalent to $1600-3200 \mathrm{mg}$ of the dried CR rhizome) yielded negative results [44, $47,51,52]$. The outcomes of these trials can be interpreted in the light of the inverted U-shaped dose-effect curve (IUSDEC) pharmacodynamic properties typical of some pharmacologically active substances, most notably D2-receptor antagonists [53].

In addition, several and diverse products with different quality standards are present on the market and the use of a low-quality product may greatly affect the outcome of a clinical trial. For instance, the analytical profiles of 11 commercial CR preparations available on the U.S. market were analyzed by means of high-performance liquid chromatography: more than one-third of them showed signs of

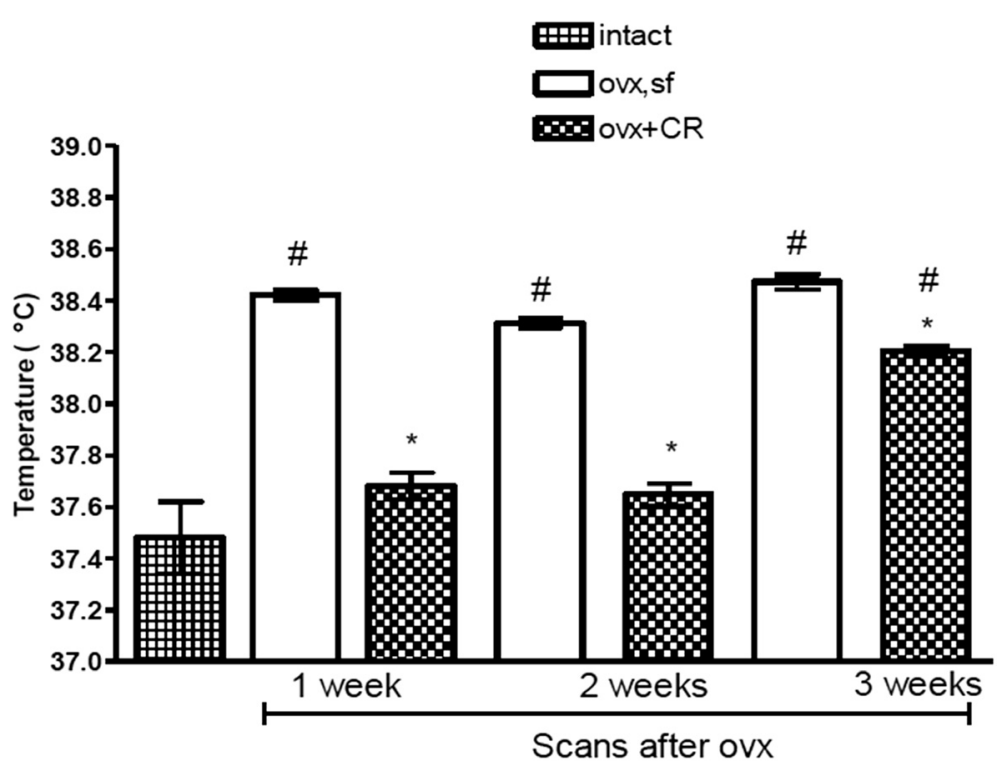

Fig. 6 Skin temperatures in untreated and CR treated rats after ovariectomy. The absence of hot flushes prior to ovx results in significantly lower skin temperature than in ovx rats. Skin temperature in the ovx CR BNO 1055 treated animals is also significantly lower than in the sham treated ovx animals ( $\# p<0.05$ vs intact; ${ }^{*} p<0.05$ vs ovx). CR: Cimicifuga racemosa; ovx: ovariectomised 
Table 1 Short description of clinical studies with Cimiciduga racemosa preparations

\begin{tabular}{|c|c|c|c|c|c|c|}
\hline $\begin{array}{l}\text { Ref. (placebo or } \\
\text { comparator controlled) }\end{array}$ & Design & $\begin{array}{l}\text { Patient } \\
\text { characteristics and } \\
\text { age }\end{array}$ & Treatment, dose, and duration & Outcome measures & Finding and comments & $\begin{array}{l}\text { Effects in short } \\
\text { No }=\varnothing \\
\text { Signif. pos }=+ \\
\text { Signif. neg }=-\end{array}$ \\
\hline Wuttke et al., 2003 [34] & $\begin{array}{l}\text { Randomized, } \\
\text { double-blind, } \\
\text { comparator and } \\
\text { placebo-controlled }\end{array}$ & $\begin{array}{l}64 \text { early } \\
\text { postmenopausal } \\
\text { women with } 3 \text { or } \\
\text { more hot flushes } \\
\text { daily }\end{array}$ & $\begin{array}{l}\text { Aquous-ethanolic CR extract BNO } 1055 \text { ( } 40 \mathrm{mg} \text { dried } \\
\text { rhizome powder daily (equivalent to } 4.5-8.9 \mathrm{mg} \\
\text { dried extract)) vs } 0.6 \mathrm{mg} \text { conjugated estrogens vs } \\
\text { placebo. Duration, } 3 \mathrm{mo}\end{array}$ & $\begin{array}{l}\text { Menopause Rating } \\
\text { Scale I. Frequency } \\
\text { of nocturnal wake- } \\
\text { up periods }\end{array}$ & $\begin{array}{l}\text { Significantly better } \\
\text { improvement of most } \\
\text { climacteric complaints under CR } \\
\text { an CE in comparison to } \\
\text { placebo, }\end{array}$ & + \\
\hline Osmers et al. 2005 [41] & $\begin{array}{l}\text { Randomized, } \\
\text { double-blind, } \\
\text { placebo-controlled }\end{array}$ & $\begin{array}{l}304 \text { early post- } \\
\text { menopausal } \\
\text { women }\end{array}$ & $\begin{array}{l}\text { Isopropanolic CR extract ( } 40 \mathrm{mg} \text { dried rhizome } \\
\text { powder daily (equivalent to } 5 \mathrm{mg} \text { dreid extract)), } \\
\text { duration } 12 \text { weeks }\end{array}$ & $\begin{array}{l}\text { Menopause Rating } \\
\text { scale I. }\end{array}$ & $\begin{array}{l}\text { Significantly better } \\
\text { improvement of most } \\
\text { climacteric complaints under CR } \\
\text { an CE in comparison to } \\
\text { placebo, }\end{array}$ & + \\
\hline Nappi et al. 2005 [42] & $\begin{array}{l}\text { Randomized, } \\
\text { comparator } \\
\text { controlled trial }\end{array}$ & $\begin{array}{l}64 \text { postmenopausal } \\
\text { women }\end{array}$ & $\begin{array}{l}\text { Isopropanolic CR extract ( } 40 \mathrm{mg} \text { dried rhizome } \\
\text { powder daily (equivalent to } 5 \mathrm{mg} \text { dried extract)) vs } \\
\text { low-dos transdermal estradiol, duration } 3 \text { months }\end{array}$ & $\begin{array}{l}\text { Diary recorded } \\
\text { number of hot } \\
\text { flushes per day }\end{array}$ & $\begin{array}{l}\text { Identical improvement under } \\
\text { CR and transdermal E2 }\end{array}$ & + \\
\hline Frei-Kleiner et al. 2005 [43] & $\begin{array}{l}\text { Randomized, } \\
\text { double-blind, } \\
\text { placebo-controlled } \\
\text { trial }\end{array}$ & $\begin{array}{l}122 \\
\text { postmenopausal } \\
\text { women with } 3 \text { or } \\
\text { more hot flushes } \\
\text { per day }\end{array}$ & CR extracr Cr 99 vs placebo, duration 12 weeks & $\begin{array}{l}\text { Kupperman Index } \\
\text { and Menopause } \\
\text { Rating Scale }\end{array}$ & $\begin{array}{l}\text { Significant superiority of the } C R \\
\text { extract in comparison to } \\
\text { placebo. }\end{array}$ & + \\
\hline \multirow[t]{2}{*}{ Pockaj et al. 2006} & \multirow{2}{*}{$\begin{array}{l}\text { Randomized, } \\
\text { double-blind, } \\
\text { placebo-controlled, } \\
\text { trial, cross-over } \\
\text { design }\end{array}$} & \multirow[t]{2}{*}{$\begin{array}{l}132 \\
\text { postmenopausal } \\
\text { women }\end{array}$} & \multirow[t]{2}{*}{$\begin{array}{l}2 \times 20 \mathrm{mg} \text { of a CR extract (probably equivalent to } \\
2 \times 200 \mathrm{mg} \text { dried root powder) for } 4 \text { weeks, then } \\
\text { crossover to verum or placebo respectively }\end{array}$} & Hot flush score & $\begin{array}{l}\text { Slightly better improvement } \\
\text { under placebo in comparison to } \\
\text { verum. }\end{array}$ & \multirow[t]{2}{*}{-} \\
\hline & & & & & $\begin{array}{l}\text { Note: The dose was } \\
\text { approximately } 10 \text {-fold higher } \\
\text { than in all other studies which } \\
\text { had a positive outcome }\end{array}$ & \\
\hline
\end{tabular}


Table 2 Short description of clinical studies with Cimiciduga racemosa preparations preparations

\begin{tabular}{|c|c|c|c|c|c|c|}
\hline $\begin{array}{l}\text { Ref. (placebo or comparator } \\
\text { controlled) }\end{array}$ & Design & $\begin{array}{l}\text { Patient } \\
\text { characteristics } \\
\text { and age }\end{array}$ & Treatment, dose, and duration & Outcome measures & Finding and comments & $\begin{array}{l}\text { Effects in short } \\
\text { No }=\varnothing \\
\text { Signif. pos }=+ \\
\text { Signif. neg }=-\end{array}$ \\
\hline \multirow[t]{2}{*}{ Newton et al. 2006 [44] } & \multirow[t]{2}{*}{$\begin{array}{l}\text { Randomized, double- } \\
\text { blind, comparator } \\
\text { and placebo- } \\
\text { controlled }\end{array}$} & \multirow[t]{2}{*}{$\begin{array}{l}351 \text { early } \\
\text { postmenopausal } \\
\text { women with } 2 \\
\text { or more hot } \\
\text { flushes daily }\end{array}$} & \multirow[t]{2}{*}{$\begin{array}{l}160 \mathrm{mg} \text { of a CR extract daily (probably } \\
\text { equivalent to } 1600 \mathrm{mg} \text { dried rhizome powder) } \\
\text { vs a multibotanical preparation vs placebo vs } \\
0.625 \mathrm{mg} \text { conjugated estrogens with or } \\
\text { without } 2 \mathrm{mg} \text { medroxyprogesterone- acetate, } \\
\text { duration } 1 \text { year }\end{array}$} & \multirow[t]{2}{*}{$\begin{array}{l}\text { Frequency and } \\
\text { intensity of } \\
\text { vasomotor } \\
\text { symptoms (Wiklund } \\
\text { Vasomotor Symptom } \\
\text { Subscale) }\end{array}$} & $\begin{array}{l}\text { Significant reduction under } \\
\text { hormone treatment. No significant } \\
\text { differences between CR and } \\
\text { placebo, worsening of symptoms in } \\
\text { multibotanical group. }\end{array}$ & \multirow[t]{2}{*}{-} \\
\hline & & & & & $\begin{array}{l}\text { Note: the } C R \text { dose was } \\
\text { approximately } 40 \text { fold higher than } \\
\text { in the studies which yielded } \\
\text { positive effects }\end{array}$ & \\
\hline Bai et al. 2007 [45] & $\begin{array}{l}\text { Randomized, double- } \\
\text { blind, comparator } \\
\text { controlled }\end{array}$ & $\begin{array}{l}244 \text { early post- } \\
\text { menopausal } \\
\text { women with } \\
\text { Kupperman } \\
\text { Index }>\text { or }=15\end{array}$ & $\begin{array}{l}\text { Isopropanolic CR extract ( } 40 \mathrm{mg} \text { dried rhizome } \\
\text { powder daily (equivalent to } 5 \mathrm{mg} \text { dried } \\
\text { extract)) vs } 2.5 \mathrm{mg} \text { tibolone, duration } 3 \text { months. }\end{array}$ & $\begin{array}{l}\text { Kupperman } \\
\text { Menopause Index }\end{array}$ & $\begin{array}{l}\text { CR preparation was as effective as } \\
\text { tibolone in reducing KMI score }\end{array}$ & + \\
\hline Oktem et al. 2007 [46] & $\begin{array}{l}\text { Randomized, } \\
\text { comparator } \\
\text { controlled trial }\end{array}$ & $\begin{array}{l}120 \text { women with } \\
\text { climacteric } \\
\text { symptoms }\end{array}$ & $\begin{array}{l}\text { Aquous-ethanolic CR extract BNO } 1055 \text { ( } 40 \mathrm{mg} \\
\text { dried rhizome powder daily (equivalent to } \\
\text { approximately } 6.5 \mathrm{mg} \text { dried extract)) vs } \\
\text { serotonin reuptake inhibitor fluoxetine, } \\
\text { duration } 6 \text { months }\end{array}$ & $\begin{array}{l}\text { Kupperman } \\
\text { Menopause Index, } \\
\text { Beck's Depression } \\
\text { Scale }\end{array}$ & $\begin{array}{l}\text { Hot flush score improved by } 85 \% \\
\text { vs } 62 \text { under fluoxetine, whereas } \\
\text { fluoxetine was more effective than } \\
\text { CR to reduce depressive symptoms }\end{array}$ & + \\
\hline \multirow[t]{2}{*}{ Geller et al. 2009 [47] } & \multirow{2}{*}{$\begin{array}{l}\text { Randomized, double- } \\
\text { blind, comparator } \\
\text { and placebo- } \\
\text { controlled }\end{array}$} & \multirow{2}{*}{$\begin{array}{l}89 \\
\text { postmenopausal } \\
\text { women with } 35 \\
\text { or more hot } \\
\text { flushes per week }\end{array}$} & \multirow{2}{*}{$\begin{array}{l}128 \mathrm{mg} \text { of an ethanolic CR extract, vs } 398 \mathrm{mg} \\
\text { of a red clover extract (containing } 120 \mathrm{mg} \\
\text { isoflavones) vs } 0.625 \mathrm{mg} \text { conjugated estrogens } \\
\text { in combination with } 2.5 \mathrm{mg} \\
\text { medroxyprogesterone -acetate vs placebo, } \\
\text { duration } 12 \text { months }\end{array}$} & \multirow{2}{*}{$\begin{array}{l}\text { Number of } \\
\text { vasomotor } \\
\text { symptoms and night } \\
\text { sweats }\end{array}$} & $\begin{array}{l}\text { No significant effects by both plant } \\
\text { preparation Significant superiority } \\
\text { of the hormone preparation. }\end{array}$ & \multirow[t]{2}{*}{-} \\
\hline & & & & & $\begin{array}{l}\text { Note: the } C R \text { dose was } \\
\text { approximately } 25 \text { fold higher than } \\
\text { in the studies which yielded } \\
\text { positive effects }\end{array}$ & \\
\hline \multirow[t]{2}{*}{ Van der Sluijs et al. 2009 [52] } & \multirow{2}{*}{$\begin{array}{l}\text { Randomized, double- } \\
\text { blind placebo con- } \\
\text { trolled trial }\end{array}$} & \multirow{2}{*}{$\begin{array}{l}93 \text { women } \\
\text { women with } 6 \\
\text { or more } \\
\text { vasomotor } \\
\text { symptoms per } \\
\text { day }\end{array}$} & \multirow{2}{*}{$\begin{array}{l}3150 \text { mg dried rhizome powder (equivalent to } \\
\text { approximately } 300 \text { mg CR extract) per day, } \\
\text { duration } 16 \text { weeks }\end{array}$} & \multirow[t]{2}{*}{$\begin{array}{l}\text { Greene Climacte5ric } \\
\text { Sclae }\end{array}$} & $\begin{array}{l}\text { No significantly more improvement } \\
\text { in comparison to placebo }\end{array}$ & \multirow[t]{2}{*}{ 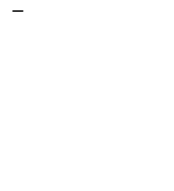 } \\
\hline & & & & & $\begin{array}{l}\text { Note: The CR dose was } \\
\text { approximately } 50 \text { fold higher than } \\
\text { in the studies which positive results }\end{array}$ & \\
\hline
\end{tabular}


Table 3 Short description of clinical studies with Cimiciduga racemosa preparations

\begin{tabular}{|c|c|c|c|c|c|c|}
\hline $\begin{array}{l}\text { Ref. (placebo or comparator } \\
\text { controlled) }\end{array}$ & Design & $\begin{array}{l}\text { Patient } \\
\text { characteristics and } \\
\text { age }\end{array}$ & Treatment, dose, and duration & Outcome measures & Finding and comments & $\begin{array}{l}\text { Effects in short } \\
\text { No }=\varnothing \\
\text { Signif. pos }=+ \\
\text { Signif. neg }=-\end{array}$ \\
\hline Rostock et al., 2001 [30] & $\begin{array}{l}\text { Prospective observational } \\
\text { trial. This non placbo } \\
\text { controlled study was } \\
\text { incorporated because of } \\
\text { the importance of its } \\
\text { outcome }\end{array}$ & $\begin{array}{l}50 \text { breast cancer } \\
\text { patients under } \\
\text { tamoxifene }\end{array}$ & $\begin{array}{l}1 \text { to } 4 \text { times } 2.5 \mathrm{mg} \text { of an } \\
\text { isopropanolic extract daily for } \\
6 \text { months }\end{array}$ & Menopause Rating Scale II & $\begin{array}{l}\text { Hot flushes, sweating, sleep } \\
\text { problems and anxiety } \\
\text { improved significantly }\end{array}$ & + \\
\hline Ross 2012 [48] & $\begin{array}{l}\text { Randomized, double-blind, } \\
\text { placebo-controlled }\end{array}$ & $\begin{array}{l}304 \text { post-meno- } \\
\text { pausal women }\end{array}$ & $\begin{array}{l}\text { Isopropanolic CR extract } \\
(40 \mathrm{mg} \text { dried rhizome powder } \\
\text { daily (equivalent to } 5 \mathrm{mg} \text { dried } \\
\text { extract)), duration } 12 \text { weeks }\end{array}$ & Number of hot flushes & $\begin{array}{l}\text { Significantly better } \\
\text { improvement of severety and } \\
\text { number of hot flushes }\end{array}$ & + \\
\hline Schellenberg et al. 2012 [49] & $\begin{array}{l}\text { Randomized, comparator } \\
\text { controlled trial }\end{array}$ & $\begin{array}{l}180 \text { women with } \\
\text { climacteric } \\
\text { complaints }\end{array}$ & $\begin{array}{l}6.5 \text { or } 13 \mathrm{mg} \text { of the CR extract } \\
\text { Ze } 450 \text { vs placebo, duration } \\
12 \text { weeks }\end{array}$ & $\begin{array}{l}\text { Kuppermans's Menopausal } \\
\text { Index. }\end{array}$ & $\begin{array}{l}\text { Significant dose dependent } \\
\text { improvement under CR }\end{array}$ & + \\
\hline $\begin{array}{l}\text { Mohammad-Alizadeh-Charandabi et al. } \\
2013 \text { [50] }\end{array}$ & $\begin{array}{l}\text { Randomized, double-blind, } \\
\text { placebo-controlled trial }\end{array}$ & $\begin{array}{l}84 \text { early } \\
\text { postmeno-pausal } \\
\text { women }\end{array}$ & $\begin{array}{l}6.5 \mathrm{mg} \text { of a dried CR extracr vs } \\
\text { placebo, duration } 8 \text { weeks }\end{array}$ & Greene Climacteric Scale & $\begin{array}{l}\text { Significant more } \\
\text { improvement by the CR } \\
\text { extract of all GCS scores } \\
\text { (vasomotor, psychiatric, } \\
\text { physical and sexual } \\
\text { symptoms) }\end{array}$ & + \\
\hline \multirow[t]{2}{*}{$\begin{array}{l}\text { Pokul and Porkhanova } 2007 \\
\text { [55]-_- } \\
\text { Stefanovskaya et al. } 2011 \text { [56] }\end{array}$} & $\begin{array}{l}\text { Prospective comparator } \\
\text { controlled trial }\end{array}$ & $\begin{array}{l}226 \text { oopher- } \\
\text { ectomized } \\
\text { women with } \\
\text { estrogen } \\
\text { dependant cancer }\end{array}$ & $\begin{array}{l}\text { Aquous-ethanolic CR extract } \\
\text { BNO } 1055 \text { ( } 40 \mathrm{mg} \text { dried } \\
\text { rhizome powder daily } \\
\text { (equivalent to approximately } \\
6.5 \mathrm{mg} \text { dried extract)) vs } \\
\text { vitamins }\end{array}$ & $\begin{array}{l}\text { Climacteric complaint } \\
\text { questionnaire, Hamilton } \\
\text { depression scale, } \\
\text { sonography of mammary } \\
\text { gland, urinary bladder } \\
\text { tonus }\end{array}$ & $\begin{array}{l}\text { Under BNO1055 significant } \\
\text { improvement of } \\
\text { psychosomatic complaints } \\
\text { and increased bladder tonus. } \\
\text { No change in mammary } \\
\text { gland }\end{array}$ & + \\
\hline & $\begin{array}{l}\text { Comparator controlled } \\
\text { study was incorporated } \\
\text { because of the importance } \\
\text { of its outcome }\end{array}$ & $\begin{array}{l}36 \text { post- } \\
\text { menopausal } \\
\text { women with } \\
\text { metabolic } \\
\text { syndrome }\end{array}$ & $\begin{array}{l}\text { Aquous-ethanolic } 6.5 \mathrm{mg} \mathrm{CR} \\
\text { extract BNO } 10556.5 \mathrm{mg} \text { vs } \\
\text { vitamins for } 6 \text { months }\end{array}$ & $\begin{array}{l}\text { Menopausal index, } \\
\text { abdominal obesity, lipids }\end{array}$ & $\begin{array}{l}\text { Reduction of psychsomatic } \\
\text { complaints and of } \\
\text { cholesterol, LDL, triglycerides } \\
\text { and fasting glucose. . } \\
\text { Increased HDL }\end{array}$ & + \\
\hline
\end{tabular}

CE conjugated oestrogens, CR Cimicifuga racemosa, DB double blind, GCS greene climacteric scale, KMI Kupperman menopause index, MRS menopause rating scale, $P M$ postmenopausal, $R C T$ randomised controlled trial 


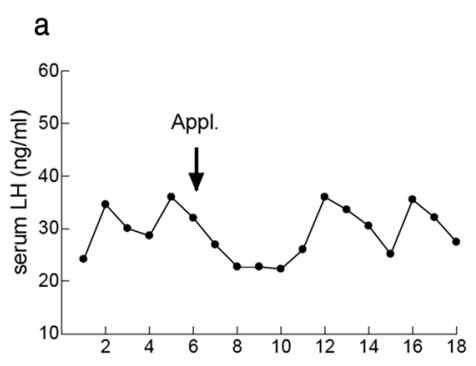

No. of blood samples (10 min intervals)

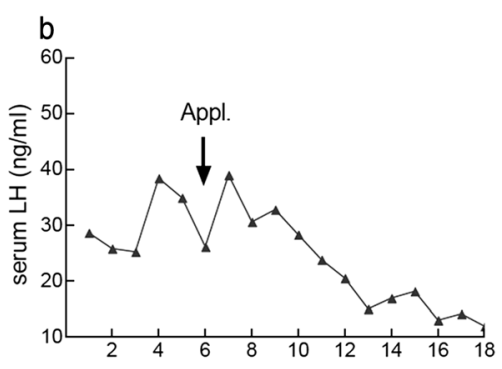

No. of blood samples (10 min intervals)

Fig. 7 Luteinizing hormone release in ovx rats. a Control, application of 2 \% Cremophor; (b) ovx rats, application of E2 $3.5 \mu$ iv. Pulsatile LH release by the pituitary occurs at the same frequency as the subcutaneously measured temperature pulses (hot flushes). This suggests a common hypothalamic mechanism that generates hot flushes and LH pulses (a). Pulsatile LH release is strongly inhibited by an injection of estradiol (b). Appl: Application; E2: Estradiol; iv: intravenous

misbranding and adulteration and most likely contained other undeclared species than C. racemosa [54].

Table 1 includes three studies that further underline the efficacy of CR extracts. In one non-controlled study in mammary cancer patients [30], climacteric complaints were successfully alleviated by a CR extract. The other 2 studies were carried out in Russia. In one of them 126 oophorectomized women suffering from heavy climacteric complaints were treated with the BNO 1055 CR extract, while 120 received conventional systemic symptomatic therapy (diuretics, tranquilizers) [55]. Substantially larger beneficial effects were seen in the CR than in the control group.

Similar results were obtained in the second study [56] that included overweight and obese patients and reported additional beneficial effects on a number of metabolic parameters, including fasting glucose and insulin resistance.

\section{CR effects on hot flushes}

In all mammals in the reproductive state so far studied, regularly occurring Gonadotropin-releasing hormone $(\mathrm{GnRH})$ pulses stimulate the pituitary gland to a normal pulsatile luteinizing hormone $(\mathrm{LH})$ release; this happens in the presence of a normally working hypothalamic $\mathrm{GnRH}$ pulse generator. In the absence of estrogens, i.e. in postmenopausal women or in ovx rats, the GnRH pulse generator is overactive. This results in high serum LH pulses and therefore in significantly higher serum gonadotropin levels as compared to those found in normal or estrogen treated individuals. In the perimenopausal state many women experience climacteric complaints, of which the hot flushes are most troublesome. The occurrence of hot flushes correlates significantly with LH pulses in the blood [57], which are the result of phasic and synchronous overactivation of hypothalamic GnRH neurons. This causes a pulsatile $\mathrm{GnRH}$ release into the portal vessels that connect the hypothalamus to the anterior pituitary gland. The overactivity of the GnRH pulse generator is due to a dysregulated neurotransmitter release. An estrogen regulated, coordinated release of serotonin, $\gamma$-amino-butyric acid (GABA) and catecholamines (dopamine, norepinephrine) in the hypothalamus is of crucial importance for the occurrence of pulsatile LH release. Ovariectomy of rats results in the typical pulsatile LH release (Fig. 7a, b) also seen in postmenopausal women whereas estradiol inhibits it [58].
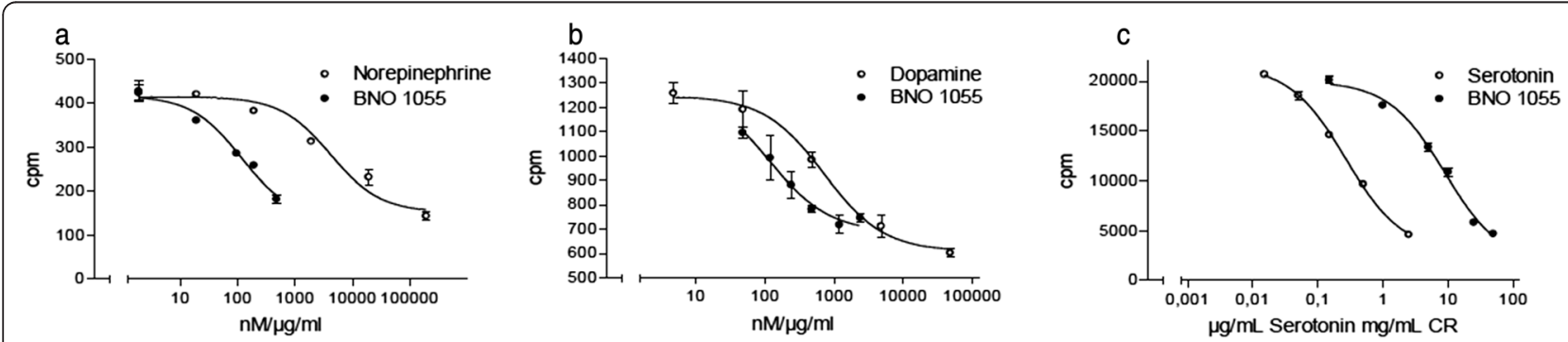

Fig. 8 Displacement curves of the BNO 1055 CR extract and the reference compounds dopamine (DA), norepinephrine (NE) or serotonin 5-HT in receptor binding assays (RBA). a Norepinephrine RBA; (b) Dopamine RBA; (c) Serotonin RBA. The receptor preparations originate from rat brain tissue. Radioactively labeled DA, NE and $5-H T$ bind to their respective receptors and substances with affinities to the respective receptor displace the radiolabeled neurotransmitter. This indicates that the BNO 1055 CR extract contains NE-, DA- and 5HT- mimetic substances. CR: Cimicifuga racemosa 

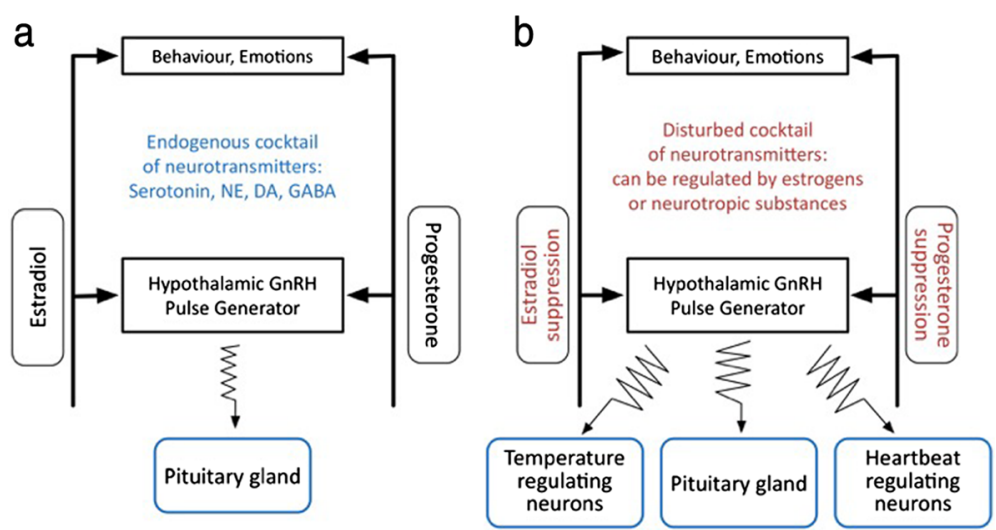

Fig. 9 Functional pictogram of the GnRH pulse generator, hot flushes and neurotransmitters. a individuals with functional ovaries such as premenopausal women or intact rodents; (b) oestrogen-deficient such as postmenopausal women or ovariectomized rodents. In the presence of functional ovaries a cocktail of stimulatory and inhibitory neurotransmitters in the hypothalamus [such as serotonin, norepinephrine (NE), dopamine (DA) and $\gamma$-aminobutyric acid (GABA)] causes regular, phasic and synchronous activation of GnRH-producing neurons, which results in normal pulsatile release of GnRH into the portal vessels connecting the hypothalamus with the anterior pituitary gland. This pulsatile pattern is important for the normal sensitivity of GnRH receptors of LH and FSH producing cells and therefore for normal reproductive health. The absence of ovarian steroids causes a dysbalance of neurotransmitters and the GnRH neurons are overstimulated, thus resulting in high GnRH pulses and consequently high LH and FSH levels in the serum. Under these conditions the dysregulated neurotransmitters spill over to temperature and heart regulating neurons, thus producing hot flushes and tachycardia

Recently we investigated whether or not the BNO 1055 CR extract contains substances that bind receptors of neurotransmitters known to be involved in the generation of hot flushes (Wuttke and Seidlová-Wuttke, unpublished). We could demonstrate that BNO 1055 contains substances that bind to dopamine, norepinephrine and serotonin (5-hydroxytryptamine: 5-HT) receptors isolated from rat brains (Fig. 8a-c). The serotoninergic substance is most likely 5-methyl-serotonin, the presence of which was already demonstrated in a CR extract [59]. In addition to dopaminergic, noradrenergic and serotoninergic substances the aqueous/ethanolic BNO 1055 CR extract contains GABAergic compounds. Clinically, GABAergic drugs such as Gabapentin and serotoninergic compounds such as the 5-HT reuptake inhibitor fluoxetine can reduce hot flushes [60]. Several triterpenes isolated from BNO 1055 were shown to bind to GABA receptors [61], hence substances with neurotransmitter like properties present in CR extracts may be at least partly responsible for the efficacy of $\mathrm{CR}$ extracts in preventing climacteric complaints. In the absence of estrogens or under conditions of blocked estrogen receptors, as under tamoxifen therapy, the release of these excitatory and inhibitory neurotransmitters appears to be exaggerated and they spill over to thermo- and cardioregulatory hypothalamic neurons, resulting in hot flushes and tachycardia attacks in many climacteric women (Fig. 9a, b).

In a 6-month clinical study in 120 climacteric patients comparing the serotonin reuptake inhibitor fluoxetine with an aqueous/ethanolic CR extract (120 patients, 6 months) the CR extract reduced $85 \%$ of the climacteric complaints as measured by the Kupperman Menopause Index, as compared to $62 \%$ in the fluoxetine group; fluoxetine, on the other hand and as expected, was more effective in reducing depression [46].

Inhibition of serotonin reuptake by neurons increases the availability of this neurotransmitter in the synaptic clefts. Mixed serotonin and norepinephrine (NE) reuptake inhibitors, which increase the availability of 5$\mathrm{HT}$ and NE, and clonidine, an agonist of the $\alpha_{2} \mathrm{NE}$ receptor, proved to be effective in reducing climacteric complaints [62]: correspondingly, the CR derived serotonin analogue 5-methyl-serotonin is probably involved in the reduction of climacteric complaints, even if the amount of 5-methyl-serotonin alone present in the daily recommended CR dosage may not be sufficient to reduce hot flushes. The efficacy of $\mathrm{CR}$ extracts in reducing climacteric complaints is most likely the result of a synergistic effect of all neurotransmitter-mimetic compounds such as the structurally identified serotoninergic and GABAergic substances $[59,61]$ and still unidentified compounds binding to dopamine and norepinephrine receptors.

\section{Common adverse events of $C R$ extracts}

In several placebo-controlled clinical trials the common adverse events which occurred under therapy with the 2 leading brands of $C R$ preparation were evaluated and are listed in Tables 4 and 5 (Osmers et al. [38]). All adverse events, regardless of the judgement of causality, are listed for the 3 studies according to their frequency of occurrence. 
Table 4 Incidence of Adverse Events /AE) in individual studies- by system organ class (MedDRA)

\begin{tabular}{|c|c|c|c|c|c|c|c|}
\hline \multirow[t]{5}{*}{ System organ class } & \multicolumn{7}{|c|}{ Reported incidence by treatment groups } \\
\hline & \multicolumn{5}{|c|}{ (KLI-DF-1 2001) } & \multicolumn{2}{|l|}{ (B| 563.2) } \\
\hline & $\begin{array}{l}\text { Klimadynon } \\
40 \mathrm{mg}\end{array}$ & $\begin{array}{l}\text { CR BNO } 1055 \\
150 \mathrm{mg}\end{array}$ & $\begin{array}{l}\text { CR BNO } 1055 \\
300 \mathrm{mg}\end{array}$ & $\begin{array}{l}\mathrm{CE} \\
0.625 \mathrm{mg}\end{array}$ & Placebo & $\begin{array}{l}\text { Menofem } \\
\text { /Klimadynon } 40 \text { mg }\end{array}$ & Placebo \\
\hline & $N=30$ & $N=33$ & $N=31$ & $N=34$ & $N=33$ & $N=152$ & $N=159$ \\
\hline & $N(\%)$ & $N(\%)$ & $N(\%)$ & $N(\%)$ & $N(\%)$ & $N(\%)$ & $N(\%)$ \\
\hline Patients with $\mathrm{AE}$ & $12(40.0)$ & $12(36.4)$ & $13(41.9)$ & $13(38.2)$ & $12(36.4)$ & $16(10.5)$ & $17(10.7)$ \\
\hline Blood and lymphatic disorders & & & & & & & $1(0.6)$ \\
\hline \multicolumn{8}{|l|}{ Cardiac disorders } \\
\hline Ear and labyrinth disorders & $1(3.3)$ & & & & $1(3.0)$ & & \\
\hline Eye disorders & & $1(3.0)$ & & & & & \\
\hline Gastrointestinal disorders & & $2(6.1)$ & & $4(11.8)$ & & $2(1.3)$ & $2(1.3)$ \\
\hline $\begin{array}{l}\text { General disorders and administration } \\
\text { site conditions }\end{array}$ & & & & & & $1(0.7)$ & $1(0.6)$ \\
\hline Immune system disorders & & & & & & & $2(1.3)$ \\
\hline Infections and infestations & $2(6.7)$ & $3(9.1)$ & $1(3.2)$ & $6(17.6)$ & & $5(3.3)$ & $4(2.5)$ \\
\hline $\begin{array}{l}\text { Injury poisoning and procedural } \\
\text { complications }\end{array}$ & $2(6.7)$ & & & & & $1(0.7)$ & \\
\hline Investigations & & $4(12.1)$ & $3(9.7)$ & $1(2.9)$ & & $1(0.7)$ & $2(1.3)$ \\
\hline Metabolism and nutrition disorders & $1(3.3)$ & $2(6.1)$ & & & $1(3.0)$ & $4(2.6)$ & $5(3.1)$ \\
\hline $\begin{array}{l}\text { Musculoskeletal and connective tissue } \\
\text { disorders }\end{array}$ & & $1(3.0)$ & $1(3.2)$ & & $2(6.1)$ & $1(0.7)$ & $2(1.3)$ \\
\hline Nervous system disorders & $1(3.3)$ & $1(3.0)$ & & & & $1(0.7)$ & $1(0.6)$ \\
\hline Psychiatric disorders & & $1(3.0)$ & & & & & \\
\hline \multicolumn{8}{|l|}{ Renal and urinary disorders } \\
\hline $\begin{array}{l}\text { Reproductive system and breast } \\
\text { disorders }\end{array}$ & $9(30.0)$ & $11(33.3)$ & $12(38.7)$ & $12(35.3)$ & $13(39.4)$ & $1(0.7)$ & $1(0.6)$ \\
\hline $\begin{array}{l}\text { Respiratory, thoracic and mediastinal } \\
\text { disorders }\end{array}$ & $1(3.3)$ & & & & $1(3.0)$ & & \\
\hline Skin and subcutaneous disorders & & & & $1(2.9)$ & $1(3.0)$ & & \\
\hline Surgical and medical procedures & & & & & & $1(0.7)$ & \\
\hline Vascular disorders & $1(3.3)$ & & & & & & $1(0.6)$ \\
\hline
\end{tabular}

With the exception of a possible influence on lipometabolism, which up to now could not definitively be excluded, no common adverse events have been identified for Cimicifuga racemosa preparations.

Several years ago case reports were published which indicated possible hepatotoxic effects of CR preparations. For this reason an expert panel was called by the US American Food and Drug Administration (Workshop on the Safety of Black Cohosh in Clinical Studies; WORKSHOP SPONSORS: National Center for Complementary and Alternative Medicine NIH Office of Dietary Supplements National Institutes of Health Bethesda, Maryland) which took place on November 22, 2004). On the basis of extensive discussion of the reported cases the expert panel concludes that $\mathrm{CR}$ extract are unlikely to be hepatotoxic. Following publication of this expert opinion an evaluation of the 30 reports on liver toxicity by black cohosh concluded that liver damage was in no case probably or certainly due to the blamed preparations. Nevertheless the Dietary Supplement Information Expert Committee determined on the basis of these data that black cohosh preparations should be labeled to include a cautionary statement [63]. Several reviews however, substantiated the safety of Cimicifuga racemosa preparations $[64,65]$. A recent meta-analysis evaluated 5 randomized, double blind and controlled studies in all of which no adverse effects on liver function were reported [66].

\section{Conclusions}

Cimicifuga racemosa extracts are traditionally used to ease climacteric complaints and a number of doubleblind placebo-controlled studies have demonstrated this effect. Low or intermediate doses, however, are more effective than high doses. CR extracts do not contain 
Table 5 Most common AE in the study by Osmers et al. (2005) [41] by system organ class (MedDRA)

\begin{tabular}{|c|c|c|c|}
\hline \multirow[t]{3}{*}{ System organ class } & $\begin{array}{l}\text { Remifemin } \\
40 \mathrm{mg}\end{array}$ & Placebo & Total \\
\hline & $N=153$ & $N=151$ & $N=304$ \\
\hline & N & N & $N(\%)$ \\
\hline Infections and infestations & 13 & 19 & $32(10.5)$ \\
\hline $\begin{array}{l}\text { Musculoskeletal and connective } \\
\text { tissue disorders }\end{array}$ & 15 & 10 & $25(8.2)$ \\
\hline Gastrointestinal disorders & 8 & 7 & $15(4.9)$ \\
\hline Investigations & 6 & 5 & $11(0.4)$ \\
\hline Nervous system disorders & 4 & 5 & $9(3.0)$ \\
\hline $\begin{array}{l}\text { Reproductive system and breast } \\
\text { disorders }\end{array}$ & 4 & 4 & $8(2.6)$ \\
\hline Psychiatric disorders & 2 & 5 & $7(2.3)$ \\
\hline Skin and subcutaneous disorders & 3 & 3 & $6(2.0)$ \\
\hline Blood and lymphatic disorders & 1 & 1 & $2(0.7)$ \\
\hline Cardiac disorders & 2 & & $2(0.7)$ \\
\hline $\begin{array}{l}\text { General disorders and administration } \\
\text { site conditions }\end{array}$ & 1 & 1 & $2(0.7)$ \\
\hline Vascular disorders & 1 & 1 & $2(0.7)$ \\
\hline $\begin{array}{l}\text { Injury poisoning and procedural } \\
\text { complications }\end{array}$ & 2 & & $2(0.7)$ \\
\hline Metabolism and nutrition disorders & 2 & & $2(0.7)$ \\
\hline Ear and labyrinth disorders & & 1 & $1(0.3)$ \\
\hline Renal and urinary disorders & 1 & & $1(0.3)$ \\
\hline $\begin{array}{l}\text { Respiratory, thoracic and mediastinal } \\
\text { disorders }\end{array}$ & & 1 & $1(0.3)$ \\
\hline
\end{tabular}

estrogenic compounds: they exert their efficacy through mechanisms linked to the presence of dopaminergic, noradrenergic, serotoninergic and GABAergic acting substances, but no estrogenic activity can be expected.

\section{Competing interests}

Wolfgang Wuttke and Dana Seidlová-Wuttke declare that they have no competing interests.

\section{Authors' contributions}

WW and DSW were advisors to Bionorica SE, Germany. They conducted the systematic review of the literature and drafted the first version of the manuscript. Both authors read and approved the present version of the manuscript. They thank Mr. Petrini for lingual help.

Received: 3 June 2015 Accepted: 5 October 2015

Published online: 20 November 2015

\section{References}

1. Rossouw JE, Anderson GL, Prentice RL, LaCroix AZ, Kooperberg C, Stefanick $\mathrm{ML}$, et al. Risks and benefits of estrogen plus progestin in healthy postmenopausal women: principal results from the Women's Health Initiative randomized controlled trial. JAMA. 2002;288(3):321-33.

2. Beral V. Breast cancer and hormone-replacement therapy in the Million Women Study. Lancet. 2003:362(9382):419-27.

3. Beral V, Reeves G, Bull D, Green J. Breast cancer risk in relation to the interval between menopause and starting hormone therapy. J Natl Cancer Inst. 2011;103(4):296-305.
4. Banks E, Beral V, Reeves G. Published results on breast cancer and hormone replacement therapy in the Million Women Study are correct. Climacteric. 2004;7(4):415-6. author reply 6-7.

5. Lyytinen H, Pukkala E, Ylikorkala O. Breast cancer risk in postmenopausal women using estradiol-progestogen therapy. Obstet Gynecol. 2009;113(1):65-73.

6. Harman SM, Vittinghoff E, Brinton EA, Budoff MJ, Cedars MI, Lobo RA, et al. Timing and duration of menopausal hormone treatment may affect cardiovascular outcomes. Am J Med. 2011;124(3):199-205.

7. Lamartiniere CA. Protection against breast cancer with genistein: a component of soy. Am J Clin Nutr. 2000;71(6 Suppl):1705S-7. discussion 8S-95.

8. Cotroneo MS, Wang J, Fritz WA, Eltoum IE, Lamartiniere CA. Genistein action in the prepubertal mammary gland in a chemoprevention model. Carcinogenesis. 2002;23(9):1467-74.

9. Wang J, Betancourt AM, Mobley JA, Lamartiniere CA. Proteomic discovery of genistein action in the rat mammary gland. J Proteome Res. 2011:10(4):1621-31.

10. Messina M, Hilakivi-Clarke L. Early intake appears to be the key to the proposed protective effects of soy intake against breast cancer. Nutr Cancer. 2009;61(6):792-8.

11. Wuttke W, Jarry H, Seidlova-Wuttke D. Isoflavones-safe food additives or dangerous drugs? Ageing Res Rev. 2007;6(2):150-88.

12. Eden JA. Phytoestrogens for menopausal symptoms: a review. Maturitas. 2012;72(2):157-9

13. Levis S, Strickman-Stein N, Ganjei-Azar P, Xu P, Doerge DR, Krischer J. Soy isoflavones in the prevention of menopausal bone loss and menopausal symptoms: a randomized, double-blind trial. Arch Intern Med. 2011;171(15):1363-9.

14. Lagari VS, Levis S. Phytoestrogens for menopausal bone loss and climacteric symptoms. J Steroid Biochem Mol Biol. 2014;139:294-301.

15. Lagari VS, Levis S. Phytoestrogens in the prevention of postmenopausal bone loss. J Clin Densitom. 2013;16(4):445-9.

16. Lagari VS, Levis S. Phytoestrogens and bone health. Curr Opin Endocrinol Diabetes Obes. 2010;17(6):546-53.

17. ESCOP. Ginseng radix. E/S/C/O/P, European Scientific Cooperative on Phytotherapy; 2003.

18. HMPC. Community herbal monograph on Cimicifuga racemosa (L.) Nutt., rhizoma. EMA/HMPC/600717/2007 Corr. 2010;25 November 2010.

19. Jarry $\mathrm{H}$, Harnischfeger $\mathrm{G}$. Studies on the endocrine effects of the contents of Cimicifuga racemosa. Planta Med. 1985;51(1):46-9.

20. Wuttke W, Jarry H, Haunschild J, Stecher G, Schuh M, Seidlova-Wuttke D. The non-estrogenic alternative for the treatment of climacteric complaints: Black cohosh (Cimicifuga or Actaea racemosa). J Steroid Biochem Mol Biol. 2014;139:302-10.

21. Bodinet C, Freudenstein J. Influence of Cimicifuga racemosa on the proliferation of estrogen receptor-positive human breast cancer cells. Breast Cancer Res Treat. 2002;76(1):1-10.

22. Stromeier $\mathrm{S}$, Petereit F, Nahrstedt A. Phenolic esters from the rhizomes of Cimicifuga racemosa do not cause proliferation effects in MCF-7 cells. Planta Med. 2005;71(6):495-500.

23. Einbond LS, Wen-Cai Y, He K, Wu HA, Cruz E, Roller M, et al. Growth inhibitory activity of extracts and compounds from Cimicifuga species on human breast cancer cells. Phytomedicine. 2008;15(6-7):504-11.

24. Rice S, Amon A, Whitehead SA. Ethanolic extracts of black cohosh (Actaea racemosa) inhibit growth and oestradiol synthesis from oestrone sulphate in breast cancer cells. Maturitas. 2007;56(4):359-67.

25. Owens $W$, Koeter HB. The OECD program to validate the rat uterotrophic bioassay: an overview. Environ Health Perspect. 2003;111(12):1527-9.

26. Rimoldi G, Christoffel J, Seidlova-Wuttke D, Jarry H, Wuttke W. Effects of chronic genistein treatment in mammary gland, uterus, and vagina. Environ Health Perspect. 2007;115 Suppl 1:62-8.

27. Freudenstein J, Dasenbrock C, Nisslein T. Lack of promotion of estrogendependent mammary gland tumors in vivo by an isopropanolic Cimicifuga racemosa extract. Cancer Res. 2002;62(12):3448-52.

28. Raus K, Brucker C, Gorkow C, Wuttke W. First-time proof of endometrial safety of the special black cohosh extract (Actaea or Cimicifuga racemosa extract) CR BNO 1055. Menopause. 2006;13(4):678-91.

29. Hirschberg AL, Edlund M, Svane G, Azavedo E, Skoog L, von Schoultz B. An isopropanolic extract of black cohosh does not increase mammographic breast density or breast cell proliferation in postmenopausal women. Menopause. 2007;14(1):89-96. 
30. Rostock M, Fischer J, Mumm A, Stammwitz U, Saller R, Bartsch HH. Black cohosh (Cimicifuga racemosa) in tamoxifen-treated breast cancer patients with climacteric complaints - a prospective observational study. Gynecol Endocrinol. 2011;27(10):844-8.

31. Rebbeck TR, Troxel AB, Norman S, Bunin GR, DeMichele A, Baumgarten M, et al. A retrospective case-control study of the use of hormone-related supplements and association with breast cancer. Int J Cancer. 2007;120(7):1523-8.

32. Ruhlen RL, Haubner J, Tracy JK, Zhu W, Ehya H, Lamberson WR, et al. Black cohosh does not exert an estrogenic effect on the breast. Nutr Cancer. 2007;59(2):269-77

33. Seidlova-Wuttke D, Jarry H, Becker T, Christoffel V, Wuttke W. Pharmacology of Cimicifuga racemosa extract BNO 1055 in rats: bone, fat and uterus. Maturitas. 2003:44 Suppl 1:S39-50.

34. Wuttke W, Seidlova-Wuttke D, Gorkow C. The Cimicifuga preparation BNO 1055 vs. conjugated estrogens in a double-blind placebo-controlled study: effects on menopause symptoms and bone markers. Maturitas. 2003:44 Suppl 1:S67-77.

35. Pan Y, Anthony MS, Binns M, Clarkson TB. A comparison of oral micronized estradiol with soy phytoestrogen effects on tail skin temperatures of ovariectomized rats. Menopause. 2001;8(3):171-4.

36. Berendsen $\mathrm{HH}$, Kloosterboer $\mathrm{HJ}$. Oestradiol and mirtazapine restore the disturbed tail-temperature of oestrogen-deficient rats. Eur J Pharmacol. 2003:482(1-3):329-33.

37. Williams H, Dacks PA, Rance NE. An improved method for recording tail skin temperature in the rat reveals changes during the estrous cycle and effects of ovarian steroids. Endocrinology. 2010;151(11):5389-94.

38. Puri P, Wuttke W, Seidlova-Wuttke D. 20-OH-ecdysone prevents hot flushes in ovariectomized rats. Planta Med. 2012;78(2):109-14.

39. Kapur P, Wuttke W, Seidlová-Wuttke D. The Cimicifuga racemosa special extract BNO 1055 prevents hot flashes in ovariectomized rats. Phytomedicine. 2010;17(11):890-4.

40. Ma X, Zhang H, Wang K, Yang L, Qin L, Bai W, et al. Effects of an isopropanolic-aqueous black cohosh extract on central body temperature of ovariectomized rats. J Ethnopharmacol. 2011;138(1):156-61.

41. Osmers R, Friede M, Liske E, Schnitker J, Freudenstein J, Henneicke-von Zepelin $\mathrm{HH}$. Efficacy and safety of isopropanolic black cohosh extract for climacteric symptoms. Obstet Gynecol. 2005;105(5 Pt 1):1074-83.

42. Nappi RE, Malavasi B, Brundu B, Facchinetti F. Efficacy of Cimicifuga racemosa on climacteric complaints: a randomized study versus low-dose transdermal estradiol. Gynecol Endocrinol. 2005;20(1):30-5.

43. Frei-Kleiner S, Schaffner W, Rahlfs WW, Bodmer C, Birkhauser M. Cimicifuga racemosa dried ethanolic extract in menopausal disorders: a double-blind placebo-controlled clinical trial. Maturitas. 2005;51(4):397-404.

44. Newton KM, Reed SD, LaCroix AZ, Grothaus LC, Ehrlich K, Guiltinan J. Treatment of vasomotor symptoms of menopause with black cohosh, multibotanicals, soy, hormone therapy, or placebo: a randomized trial. Ann Intern Med. 2006;145(12):869-79.

45. Bai W, Henneicke-von Zepelin HH, Wang S, Zheng S, Liu J, Zhang Z, et al. Efficacy and tolerability of a medicinal product containing an isopropanolic black cohosh extract in Chinese women with menopausal symptoms: a randomized, double blind, parallel-controlled study versus tibolone. Maturitas. 2007:58(1):31-41.

46. Oktem M, Eroglu D, Karahan HB, Taskintuna N, Kuscu E, Zeyneloglu HB. Black cohosh and fluoxetine in the treatment of postmenopausal symptoms: a prospective, randomized trial. Adv Ther. 2007;24(2):448-61.

47. Geller SE, Shulman LP, van Breemen RB, Banuvar S, Zhou Y, Epstein G, et al. Safety and efficacy of black cohosh and red clover for the management of vasomotor symptoms: a randomized controlled trial. Menopause. 2009;16(6):1156-66.

48. Ross SM. Menopause: a standardized isopropanolic black cohosh extract (remifemin) is found to be safe and effective for menopausal symptoms. Holist Nurs Pract. 2012;26(1):58-61.

49. Schellenberg R, Saller R, Hess L, Melzer J, Zimmermann C, Drewe J, et al. Dose-dependent effects of the Cimicifuga racemosa extract Ze 450 in the treatment of climacteric complaints: a randomized, placebo-controlled study. Evid Based Complement Alternat Med. 2012;2012:260301.

50. Mohammad-Alizadeh-Charandabi S, Shahnazi M, Nahaee J, Bayatipayan S. Efficacy of black cohosh (Cimicifuga racemosa L.) in treating early symptoms of menopause: a randomized clinical trial. Chin Med. 2013;8(1):20
51. Pockaj BA, Gallagher JG, Loprinzi CL, Stella PJ, Barton DL, Sloan JA, et al. Phase III double-blind, randomized, placebo-controlled crossover trial of black cohosh in the management of hot flashes: NCCTG Trial N01CC1. J Clin Oncol. 2006:24(18):2836-41.

52. van der Sluijs CP, Bensoussan A, Chang S, Baber R. A randomized placebocontrolled trial on the effectiveness of an herbal formula to alleviate menopausal vasomotor symptoms. Menopause. 2009;16(2):336-44.

53. Monte-Silva K, Kuo MF, Thirugnanasambandam N, Liebetanz D, Paulus W, Nitsche MA. Dose-dependent inverted U-shaped effect of dopamine (D2-like) receptor activation on focal and nonfocal plasticity in humans. J Neurosci. 2009;29(19):6124-31.

54. Jiang B, Kronenberg F, Nuntanakorn P, Qiu MH, Kennelly EJ. Evaluation of the botanical authenticity and phytochemical profile of black cohosh products by high-performance liquid chromatography with selected ion monitoring liquid chromatography-mass spectrometry. J Agric Food Chem. 2006;54(9):3242-53.

55. Pokul L, Porkhanova N. Clinical assessment of the use of Klimadynon in oncological patients (An analysis of the results of experimental observations). "Vrach". Journal (wwwrusvrachru). 2007;8:67-71.

56. Stefanovskaya OV, Akker LV, Galchenko Al. Experience with alternative therapy in females with menopausal metabolic syndrome. "Mother and Child in Kuzbass", special edition 2011;1:309-12.

57. Tataryn IV, Meldrum DR, Lu KH, Frumar AM, Judd HL. LH, FSH and skin temperaure during the menopausal hot flash. J Clin Endocrinol Metab. 1979;49(1):152-4.

58. Seidlova-Wuttke $D$, Hesse $O$, Jarry H, Christoffel V, Spengler B, Becker $T$, et al. Evidence for selective estrogen receptor modulator activity in a black cohosh (Cimicifuga racemosa) extract: comparison with estradiol-17beta. Eur J Endocrinol. 2003;149(4):351-62.

59. Powell SL, Godecke T, Nikolic D, Chen SN, Ahn S, Dietz B, et al. In vitro serotonergic activity of black cohosh and identification of N(omega)methylserotonin as a potential active constituent. J Agric Food Chem. 2008;56(24):11718-26.

60. Sideras K, Loprinzi CL. Nonhormonal management of hot flashes for women on risk reduction therapy. J Natl Compr Canc Netw. 2010;8(10):1171-9.

61. Cicek SS, Khom S, Taferner B, Hering S, Stuppner H. Bioactivity-guided isolation of $G A B A(A)$ receptor modulating constituents from the rhizomes of Actaea racemosa. J Nat Prod. 2010;73(12):2024-8.

62. Umland $\mathrm{EM}$, Falconieri L. Treatment options for vasomotor symptoms in menopause: focus on desvenlafaxine. Int J Womens Health. 2012;4:305-19.

63. Mahady GB, Low Dog T, Barrett ML, Chavez ML, Gardiner P, Ko R, et al. United States Pharmacopeia review of the black cohosh case reports of hepatotoxicity. Menopause. 2008;15:628-38.

64. Teschke R, Schwarzenboeck A, Schmidt-Taenzer W, Wolff A, Hennermann KH. Herb induced liver injury presumably caused by black cohosh: a survey of initially purported cases and herbal quality specifications. Ann Hepatol. 2011;10:249-59.

65. Teschke R, Schwarzenboeck A. Suspected hepatotoxicity by Cimicifugae racemosae rhizoma (black cohosh, root): critical analysis and structured causality assessment. Phytomedicine. 2009;16:72-84.

66. Naser B, Schnitker J, Minkin MJ, et al. Suspected black cohosh hepatotoxicity: no evidence by meta-analysis of randomized controlled clinical trials for isopropanolic black cohosh extract. Menopause. 2011;18:366-75.

\section{Submit your manuscript to a SpringerOpen ${ }^{\odot}$ journal and benefit from:}

- Convenient online submission

- Rigorous peer review

- Immediate publication on acceptance

- Open access: articles freely available online

- High visibility within the field

- Retaining the copyright to your article

Submit your next manuscript at $>$ springeropen.com 